\title{
Sudden visual loss associated with clostridial bacteraemia
}

\author{
ANTHONY J CANNISTRA,' DANIEL M ALBERT, 'DONALD A FRAMBACH,' \\ ROBERT J DREHER, ${ }^{2}$ AND LLOYD ROBERTS ${ }^{2}$ \\ From the 'David G Cogan Eye Pathology Laboratory, Massachusetts Eye and Ear Infirmary, Boston, \\ Massachusetts, and the ${ }^{2}$ Penobscot Bay Medical Center, Rockland, Maine, USA
}

SUMmaRY A patient with endogenously acquired Clostridium septicum panophthalmitis is presented. The patient exhibited a striking sequence of signs and symptoms associated with this devastating ocular infection. Intensive antibiotic therapy was ineffective and enucleation of the globe was required. The microscopic pathology of the enucleated globe showed extensive infarction and necrosis of ocular structures in association with the panophthalmitis. In addition thrombosis of the central retinal artery and of choroidal vessels was observed.

Clostridial ocular infections usually result from penetrating injuries of the globe, and the offending species is typically Clostridium perfringens. ${ }^{1}$ There have been only two previously reported cases where acute endophthalmitis has occurred secondarily to clostridial sepsis. ${ }^{23}$ This paper describes a case of clostridial panophthalmitis occurring subsequent to an endogenous clostridial infection, and discusses possible mechanisms of the ocular pathology observed. It is presented to make ophthalmologists aware of the occurrence of clinical and pathological findings associated with a clostridial ocular infection following systemic clostridial sepsis.

\section{Case report}

A 77-year-old white male physician was admitted to Penobscot Bay Medical Center on 1 February 1986 with a 24-hour history of fever, chills, and right upper quadrant pain. He reported acute, bloody diarrhoea 10 days prior to admission which resolved without antibiotic treatment. Stool examination at that time revealed $1+$ white blood cells, but cultures were negative for enteric pathogens. He had a history of emphysema and chronic atrial fibrillation, adenocarcinoma of the prostate gland, thought to have been cured by transurethral resection in 1985, and chronic constipation, but was otherwise in good health. He had continued to practise medicine until this present illness.

Correspondence to Daniel M Albert, MD, David G Cogan Eye Pathology Laboratory, Massachusetts Eye and Ear Infirmary, 243 Charles Street, Boston, Massachusetts 02114, USA.
Physical examination revealed a Caucasian male appearing acutely ill with abdominal pain, mild diaphoresis, and chills. Vital signs on admission were blood pressure $150 / 68 \mathrm{mmHg}$, pulse $100 /$ minute and regular, respiratory rate $32 /$ minute, and temperature $38.9^{\circ} \mathrm{C}$. Eye examination by his internist at this time showed the pupils to be equal and reactive to light, and a funduscopic examination showed nothing abnormal. On cardiovascular examination a grade $3 / 6$ systolic murmur was heard. The abdominal examination revealed decreased bowel sounds, with diffuse tenderness, especially in the right upper quadrant. There was no rebound or guarding tenderness. On rectal examination no masses were felt. Occult blood was detected in the stools. The remainder of the physical examination was unremarkable.

Laboratory data on admission showed a white cell count of $23.7 \times 10^{9} / 1$, with 88 polymorphonuclear cells and 10 band forms per 100 cells. The haemoglobin was $13.1 \mathrm{~g} / \mathrm{dl}$ and the packed cell volume (haematocrit) was $0 \cdot 37$. Other significant values include a blood urea nitrogen level of $24 \mathrm{mg} / \mathrm{dl}(4 \cdot 0$ $\mathrm{mmol} / \mathrm{l})$, creatinine of $1.3 \mathrm{mg} / \mathrm{dl}(115 \mu \mathrm{mol} / \mathrm{l})$, and a decreased total protein and albumin of $5.2 \mathrm{~g} / \mathrm{dl}(52$ $\mathrm{g} / \mathrm{l})$ and $2.9 \mathrm{~g} / \mathrm{dl}(29 \mathrm{~g} / \mathrm{l})$, respectively. Radiological examination included chest, anteroposterior plane film of the abdomen, biliary tree scan, and intravenous pyelogram. All these studies were within normal limits. A peritoneal lavage was negative for red blood cells. The differential diagnosis at this time included subacute bacterial endocarditis, pneumonia, or a primary abdominal process such as 
cholecystitis, perforation of a viscus from diverticulitis, or malignancy. Blood cultures were obtained, and intravenous clindamycin and gentamicin were begun.

Six hours after admission to hospital the patient complained of left periorbital pain and reported sudden and complete visual loss in his left eye. An ophthalmic consultation was promptly obtained. Ocular examination at this time revealed no light perception with the left eye. The right eye was entirely normal. External eye examination was significant for marked left eyelid and orbital swelling. The conjunctiva of the left eye was injected with $2+$ chemosis and a mucopurulent drainage was present. The cornea had stromal and epithelial oedema. The iris colour was blue-grey bilaterally. The left pupil was larger than the right $(2.5 \mathrm{~mm}$ compared with 1.5 $\mathrm{mm})$ and did not respond to either direct or consensual stimulation. Details of the fundus could not be seen in the left eye by either direct or indirect ophthalmoscopy. Extraocular movements were severely restricted in all directions. The tension by Schiøtz tonometry was $60 \mathrm{mmHg}$ in the left eye.

Left orbital aspiration was attempted but no fluid was obtained. The patient received topical pilocarpine, Propine (dipirefrim $\mathrm{HCl} 0 \cdot 1 \%$ ), and timolol, as well as intravenous acetazolamide. Ocular examination three hours later revealed a tension of 30 $\mathrm{mmHg}$ in the left eye, and the iris colour had now changed from blue-grey to reddish brown. Slit-lamp examination, which was initially deferred owing to the patient's poor condition, was performed eight hours after the onset of blindness. It showed a left anterior chamber that contained large sections of both pigmented material and white, flocculent material $(0.25 \mathrm{~mm}$ in diameter) suspended in a gel which occupied approximately $90 \%$ of the anterior chamber. A diagnosis of panophthalmitis and orbital cellulitis was made, and the patient was put on topical gentamicin in addition to systemic antibiotics.

On the third day in hospital the patient developed a corneal ulcer. Blood cultures obtained on admission grew Clostridium septicum, and antibiotic therapy was changed to intravenous oxacillin and chloramphenicol, and subconjunctival gentamicin was administered. On the fifth day in hospital a corneal perforation was noted and the eye was enucleated next day (Fig. 1). A significant finding at enucleation was the ischaemic appearance of the extraocular muscles. Later that evening the patient developed bilateral pleural effusions. His clinical condition gradually improved, yet he died on the thirteenth hospital day of an apparent cardiac arrest.

The cause of death at necropsy was determined to be a rupture of a dissecting lower thoracic aortic aneurysm with exsanguination into the left pleural

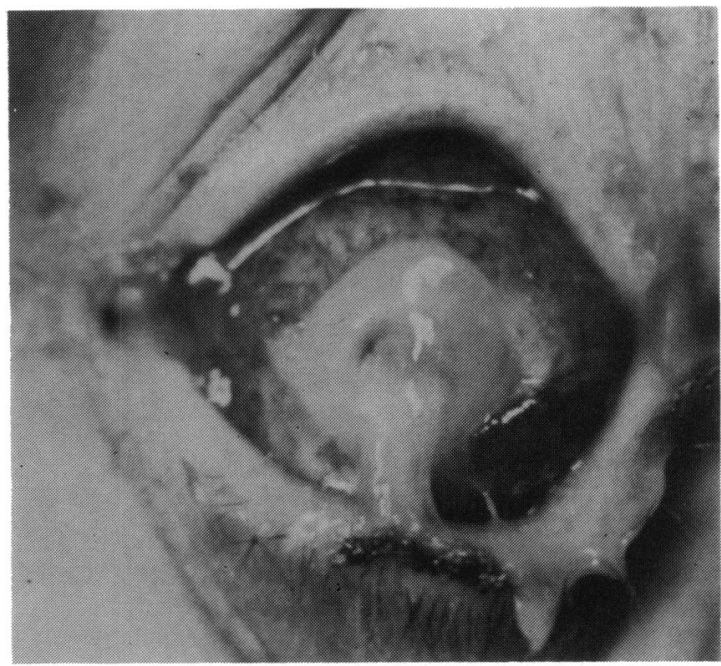

Fig. 1 Clinical photograph of left eye with central corneal perforation just prior to enucleation.

space. The rupture occurred $5 \mathrm{~cm}$ above the diaphragm, and was surrounded by a $1-2 \mathrm{~cm}$ area of mottled, organising thrombus. There was also an area of dissection in the abdominal aorta near the origin of the inferior mesenteric artery. Microscopic sections of both of these aortic regions showed extensive atheromatous plaque formation, with acute and chronic inflammation adjacent to areas of dissection. Within these areas were foci of degenerating polymorphonuclear leucocytes, suggesting recent bacterial infection. Examination of the heart revealed calcification and moderate narrowing of the coronary vasculature. Internal examination showed normal myocardium and valve structure except for a $5-6 \mathrm{~mm}$ area of pink speckling on the posterior aortic valve leaflet. Microscopic examination of this speckled area revealed extravasation of erythrocytes in the intimal and subintimal connective tissue. Cross-sections of the entire length of the ophthalmic arteries from the internal carotids to the globe on the right, and to the point of enucleation of the left globe showed no thrombus formation or inflammation. The lumen of the left internal carotid was reduced by one-half by a calcified, atherosclerotic plaque (Fig. 2). There was no evidence of meningitis, cerebral ischaemia, or thrombosis of cerebral vessels. Examination of the visceral structures was remarkable only for numerous diverticula in the sigmoid colon.

The left globe measured $21 \mathrm{~mm}$ vertically by $20 \mathrm{~mm}$ horizontally by $24 \mathrm{~mm}$ axially and had $5 \mathrm{~mm}$ of attached optic nerve. The cornea measured $11 \mathrm{~mm}$ by $10 \mathrm{~mm}$ and was opaque with a central perforation $4 \mathrm{~mm}$ in diameter. The pupil was not visible and the 


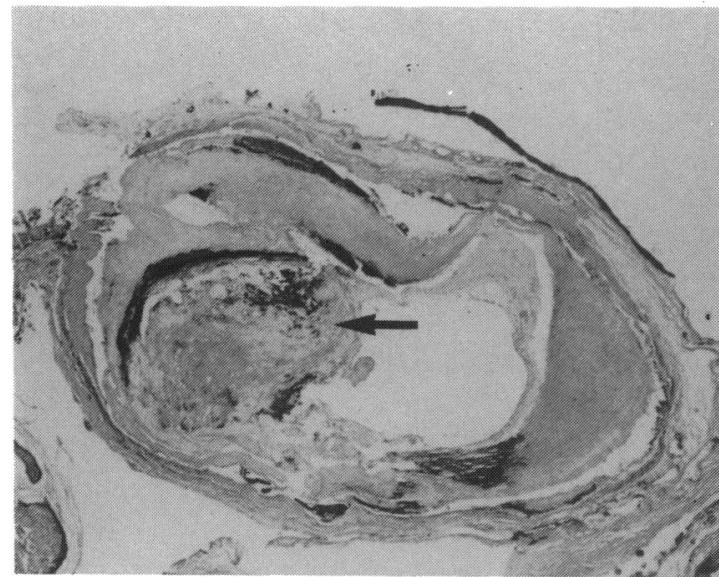

Fig. 2 Cross-section of left internal carotid artery with diameter reduced to one-half by calcified, atheromatous plaque (arrow). Haematoxylin and eosin, $\times 5$.

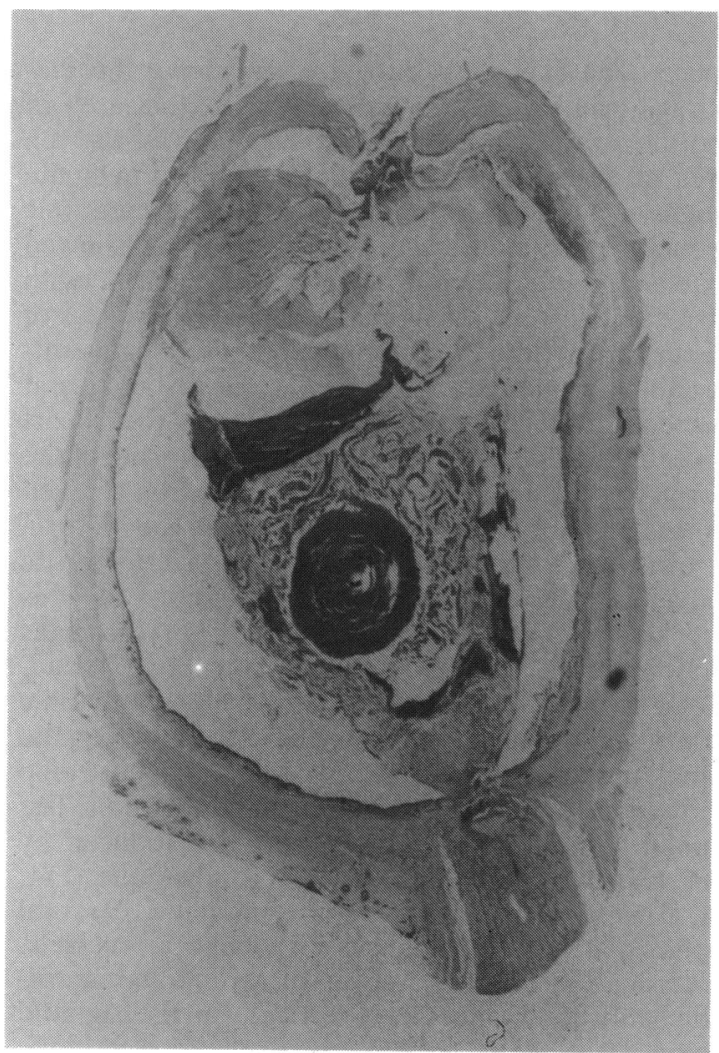

Fig. 3 Sagittal section of left eye showing severe panophthalmitis. Full-thickness perforation of central cornea, and thick inflammatory membrane behind the cornea; extensive anterior synechiae are present. The iris and ciliary body are necrotic, as is the retina, which is completely detached. Haematoxylin and eosin, $\times 3$.

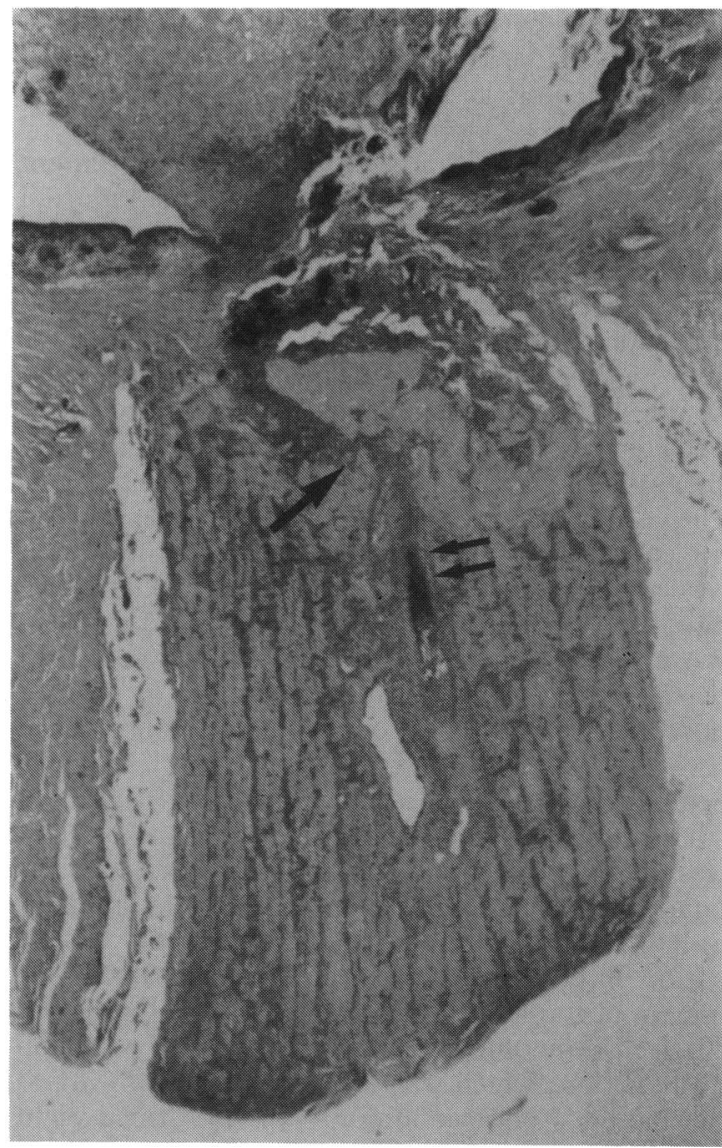

Fig. 4 Sagittal section of left optic nerve showing an area of central necrosis surrounded by acute inflammation (arrow). An occlusion of the central retinal artery by an organising thrombus is also present (arrows). Haematoxylin and eosin, $\times 1 \cdot 7$.

eye did not transilluminate light. On horizontal section the anterior chamber was obliterated and the angle was not visible. The iris, ciliary body, and lens were surrounded by white, flocculent material. The lens was dislocated posteriorly. A haemorrhagic mass filled the vitreous cavity. The retina was totally detached, and the optic disc not visible.

Microscopically there was a large, full-thickness perforation of the central cornea. The eye showed severe panophthalmitis (Fig. 3). The cornea, where present, was covered by a thin layer of epithelium with identifiable Bowmans's membrane. The corneal stroma was infiltrated with polymorphonuclear leucocytes. The residual Descemet's membrane was devoid of endothelium. An inflammatory membrane was present behind the cornea and extensive anterior synechiae were seen. The nasal portion of the iris and ciliary body were necrotic, though the inflammatory 


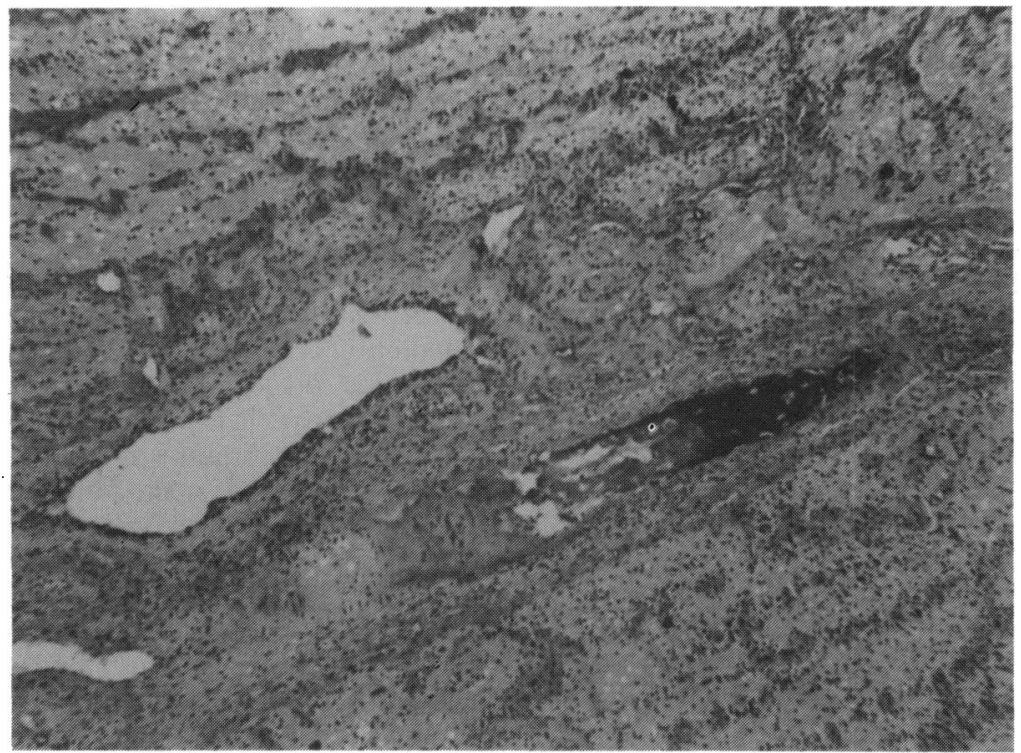

Fig. 5 Higher magnification of sagittal section through the left optic nerve. The central retinal artery and vein are shown, and the occlusion of the central retinal artery is present. Also evident is the acute inflammatory response involving the left optic nerve. Haematoxylin and eosin, $\times 16$.

response was most severe in the anterior temporal portion of the eye. The retina was completely detached and necrotic (Fig. 3). Within the optic nerve head was an area of central necrosis surrounded by acute inflammation (Fig. 4). An occlusion of the central retinal artery by an organising thrombus (Figs. 4, 5), and numerous thrombi within the choroidal vasculature were seen. Brown and Brenn stain for Gram-positive and Gram-negative bacteria (Fig. 6) and periodic acid-Schiff stain revealed numerous, large Gram-positive rods in areas of the iris, ciliary body, retina, and optic nerve. Kinyoun's acid fast stain was negative for acid-fast bacilli.

The following diagnoses were made: (1) panophthalmitis secondary to Clostridium septicum; (2) corneal perforation; (3) thrombi of the central retinal artery and choroidal vessels.

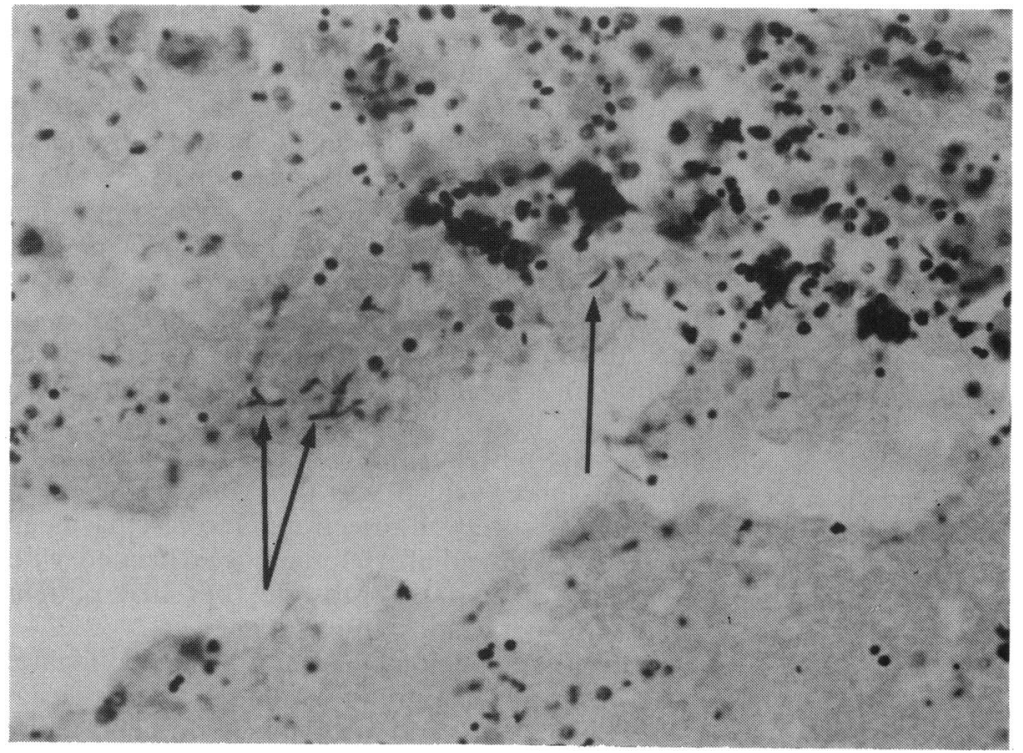

Fig. 6 High magnification of necrotic retina with several large, spindle-shaped, Gram-positive bacilli present (arrows). Brown and Brenn stain, $\times 100$. 


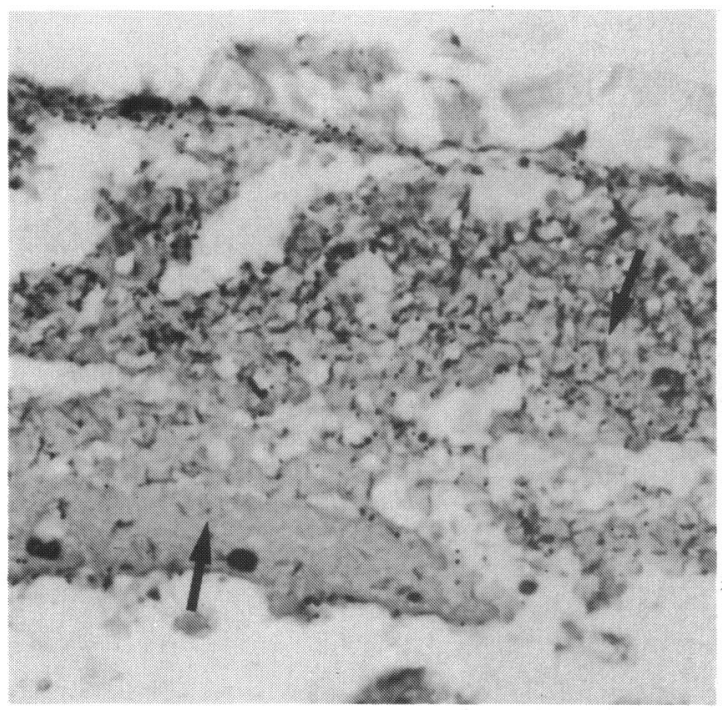

Fig. 7 Periodic acid-Schiff stain of necrotic retina showing an abundance of positively staining, spindle-shaped rod forms (arrows). $\times 10$.

\section{Discussion}

Gas gangrene panophthalmitis, characterised by Leavelle,' typically occurs after penetrating injuries to the globe with subsequent infection of the damaged tissue of Clostridium perfingens. This paper describes a patient who developed a panophthalmitis secondary to clostridial septicaemia. The diagnosis of clostridial panophthalmitis was based on the clinical evolution, the gross and histological appearance, and the presence of Clostridium septicum detected by anaerobic blood cultures prior to antibiotic treatment. An intraocular culture obtained after antibiotic treatment showed no growth of Clostridium septicum, but Gram stains and periodic acid-Schiff stains showed an abundance of Gram-positive, spindle-shaped rods in necrotic areas of iris, ciliary body, retina, and optic nerve. These morphological findings, together with positive blood cultures for Clostridium septicum, indicate that this organism was responsible for the fulminant ocular destruction observed in this patient.

A search of the literature reveals only two cases similar to that now described. One patient was a 68year-old man who had a perforated gangrenous gall bladder and Clostridium perfringens sepsis. ${ }^{2}$ The second was a 78-year-old woman with acute gastroenteritis and Clostridium septicum sepsis. ${ }^{3}$ The patient reported here had many of the typical symptoms of clostridial endophthalmitis. ${ }^{1}$ He experienced sudden left orbital pain and visual loss accompanied by oedema of the lids and periorbital tissue. In addition a raised intraocular pressure, conjunctival chemosis with a change of iris colour to reddish brown, and eventual corneal ulceration and perforation were observed. No gas bubble in the anterior chamber was present, a finding which has often been described in clostridial panophthalmitis. ${ }^{1-3}$

This patient's clinical history illustrates the possible source of clostridial sepsis and mechanisms responsible for the evolution of the panophthalmitis. Although no certain source of Clostridium septicum was discovered during necropsy, it is reasonable to speculate that it came from the gastrointestinal tract. The patient suffered an episode of acute, bloody diarrhoea 10 days before admission to hospital and had no recent history of antibiotic use or ulcerative colitis.

The finding of generalised atherosclerosis at necropsy and the unusual involvement of the abdominal aorta in the region of the inferior mesenteric artery suggest that colonic ischaemia may have been the cause of the bloody diarrhoea. Although the possibility of embolism in a bowel vessel exists in the setting of chronic atrial fibrillation, bowel ischaemia is usually produced by a nonocclusive event. ${ }^{4}$ In fact, approximately one-third of patients with mesenteric ischaemia do not have an identifiable occlusion of a major vessel. ${ }^{4}$ Although rectal examination was positive for occult blood in the stools, colonoscopy performed when the patient was in hospital did not show any ulcerations, friability, or bulging folds from submucosal haemorrhage. The finding of multiple sigmoidal diverticula on necropsy raises the possibility that a temporary communication between mucosal vasculature and colonic contents provided a suitable opportunity for the entry of colonic organisms into the bloodstream. This is the presumed mechanism of this patient's attack of bloody diarrhoea and subsequent development of Clostridium septicum bacteraemia.

Koransky et al. ${ }^{5}$ reported an association between Clostridium septicum bacteraemia and malignancy. In their review of 21 patients with solid tumours $67 \%$ had cancer of the colon and one patient developed cancer of the caecum two years after an episode of Clostridium septicum bacteraemia. In our patient no evidence of a gastrointestinal malignancy was found at necropsy; however, he had a history of prostatic adenocarcinoma which was thought to have been cured by transurethral resection. One case has been reported of clostridial bacteraemia associated with adenocarcinoma of the prostate, but that patient had disease metastatic to the abdomen. ${ }^{6}$

Clostridium septicum is a Gram-positive, obligate, anaerobic rod which produces four exotoxins designated alpha, beta, gamma, and delta. ${ }^{7}$ The alpha 
toxin is a lecithinase that produces the fulminant tissue necrosis and small blood vessel thrombosis seen in Clostridium septicum infections. ${ }^{7}$ The change in iris colour observed in this patient is consistent with tissue necrosis, which was confirmed on pathological examination.

Although the normal human eye has a rich blood supply, it contains several avascular structures, including the outer retina, vitreous, lens, and cornea ${ }^{8}$ Since many of the ocular vessels, including the central retinal artery, lack anastomoses, under pathological conditions the blood supply of the eye can be severely compromised. With these anatomical features in mind, it is understandable that, if a part of the ocular vasculature were interrupted either by a local toxic effect or by a distant embolic phenomenon, then the minimal collateral circulation might not be sufficient to prevent the ischaemic conditions required for the growth of Clostridium septicum.

The mechanism of sudden visual loss experienced by this patient almost certainly involves a vascular event. There was no convincing evidence of embolic foci, nor were there any systemic signs of embolisation affecting other organ systems based on necropsy findings. These observations make it unlikely that a distant embolic phenomenon was responsible for the pathology seen. It is more likely that a local ocular event involving clostridial seeding, production of exotoxin, and subsequent vessel thrombosis and tissue destruction accounts for the ocular pathology observed.

Drs Charles M Gluck and Robert P Laurence, of the Penobscot Bay Medical Center, played primary roles in the care of this patient and kindly reviewed the manuscript.

\section{References}

1 Leavelle RE. Gas gangrene panophthalmitis. Arch Ophthalmol 1955; 53: 634-42.

2 Frantz JM, Lemp MA, Font RL, Stone R, Eisner E. Acute endogenous panophthalmitis caused by Clostridium perfringens. Am J Ophthalmol 1974; 78: 295-303.

3 Insler MS, Karcioglu ZA, Naugle T Jr. Clostridium septicum panophthalmitis with systemic complications. Br J Ophthalmol 1985; 69: 774-7.

4 Renton CJ. Nonocclusive intestinal infarction. Clin Gastroenterol 1972; 3: 655-73.

5 Koransky JR, Stargel MD, Dowell VR Jr. Clostridium septicum bacteremia. Am J Med 1979; 66: 63-6.

6 Cabrera A, Tsukada Y, Pickren JW. Clostridial gas gangrene and septicemia in malignant disease. Cancer 1965; 18: 800-6.

7 Smith LD. The pathogenic anaerobic bacteria. Springfield: Thomas, 1966: 341-432.

8 Wolff E. Anatomy of the eye and orbit. 6th ed. London: Lewis, 1968: 146-9, 406-9.

Accepted for publication 20 March 1987. 